\title{
Taxonomic Identification Below Species Level
}

National Cancer Institute

\section{Source}

National Cancer Institute. Taxonomic Identification Below Species Level. NCI Thesaurus.

Code C132419.

The determination of the taxonomic ranking of the microorg anism that is below species level. 